\title{
Calculation of critical exponents by self-similar factor approximants
}

\section{Journal Article}

\section{Author(s):}

Yukalov, V.I.; Yukalova, E.P.

Publication date:

2007-01

Permanent link:

https://doi.org/10.3929/ethz-b-000068086

Rights / license:

In Copyright - Non-Commercial Use Permitted

Originally published in:

The European Physical Journal B 55(1), https://doi.org/10.1140/epjb/e2007-00044-4 


\title{
Calculation of critical exponents by self-similar factor approximants
}

\author{
V.I. Yukalov ${ }^{1,2, a}$ and E.P. Yukalova ${ }^{3}$ \\ 1 Bogolubov Laboratory of Theoretical Physics, Joint Institute for Nuclear Research, Dubna 141980, Russia \\ 2 Department of Technology and Economics, Swiss Federal Institute of Technology, Zürich 8032, Switzerland \\ 3 Laboratory of Information Technologies, Joint Institute for Nuclear Research, Dubna 141980, Russia
}

Received 20 November 2006

Published online 9 February 2007 - C EDP Sciences, Società Italiana di Fisica, Springer-Verlag 2007

\begin{abstract}
The method of self-similar factor approximants is applied to calculating the critical exponents of the $O(N)$-symmetric $\varphi^{4}$ theory and of the Ising glass. It is demonstrated that this method, being much simpler than other known techniques of series summation in calculating the critical exponents, at the same time, yields the results that are in very good agreement with those of other rather complicated numerical methods. The principal advantage of the method of self-similar factor approximants is the combination of its extraordinary simplicity and high accuracy.
\end{abstract}

PACS. 05.70.Jk Critical point phenomena - 02.30.Lt Sequences, series, and summability - 02.30.Mv Approximations and expansions

\section{Introduction}

The knowledge of critical exponents, characterizing critical phenomena, provides us with basic information on the behavior of thermodynamic quantities in the vicinity of critical points [1-3]. This is why so much efforts have been devoted to the experimental measurements as well as to theoretical calculations of these exponents.

Because of the complexity of realistic theoretical models, critical exponents can usually be represented by power series obtained with the help of some perturbation theory. Such series are practically always divergent, which requires to use resummation techniques allowing for the determination of effective limits of divergent series. The standard approach applied to the summation of series, associated with critical indices, is based on the PadéBorel-Leroy transformation and its variants [4]. Another approach is based on optimized perturbation theory [5], where the resummation is due to control functions transforming divergent series into converging ones. Introducing control functions through the change of expansion variables, it is possible to resum the field-theoretic expansions for critical exponents $[6,7]$. The convergence of the optimized perturbation theory can be greatly accelerated by invoking the self-similar approximation theory [8-16], as has been done for calculating critical exponents [17]. All these approaches, mentioned above, require quite complicated numerical calculations. A purely numerical pro-

\footnotetext{
${ }^{a}$ e-mail: yukalov@if.sc.usp.br
}

cedure of calculating critical exponents is due to Monte Carlo simulations [18-29].

In the present paper, we suggest a new approach for the summation of series related to critical exponents. This approach uses the method of self-similar factor approximants [30-33], whose mathematical foundation is based on the self-similar approximation theory [8-16]. The construction of the factor approximants is very simple and straightforward. We recall the main definitions in Section 2. Then, in Section 3, we apply these approximants for the summation of the $\varepsilon$-expansions for the critical exponents of the $N$-vector $\varphi^{4}$ field theory. Our very simple method yields the results that are in perfect agreement with the most complicated numerical procedures. In Section 4, we demonstrate that the suggested method is applicable even for such a notoriously difficult problem as finding the critical indices from the high-temperature series expansions for spin glasses. Finally, Section 5 is conclusion.

\section{Self-similar factor approximants}

Suppose that our aim is to reconstruct a real function $f(x)$ of a real variable $x$, when the function is represented by its asymptotic expansion at $x \rightarrow 0$ as a power series

$$
f_{k}(x)=\sum_{n=0}^{k} a_{n} x^{n},
$$


where $k=0,1,2, \ldots$ Without the loss of generality, we may assume that $a_{0}=1$ in expansion (1). This is because if instead of form (1) we would have a more general expression

$$
f^{(k)}=f^{(0)}(x) \sum_{n=0}^{k} a_{n}^{\prime} x^{n},
$$

with a given function $f^{(0)}(x)$, then we could return to expansion (1), with $a_{0}=1$, by defining

$$
f_{k}(x) \equiv \frac{f^{(k)}(x)}{f^{(0)}(x) a_{0}^{\prime}} .
$$

The sequence $\left\{f_{k}(x)\right\}$ is usually divergent for any finite $x$.

The method of self-similar factor approximants [3033] makes it possible to extrapolate the asymptotic expansion (1), valid only for $x \rightarrow 0$, to the whole region of the variable $x \geq 0$. When $k=2 p$ is even, with $p$ being an integer, then the even-order factor approximant is

$$
f_{2 p}^{*}(x)=\prod_{i=1}^{p}\left(1+A_{i} x\right)^{n_{i}} .
$$

The parameters $A_{i}$ and $n_{i}$ are obtained from the reexpansion procedure, that is, by expanding approximant (2) in powers of $x$ up to the $k$ th order and equating the latter expansion with the initial one given by form (1). This re-expansion procedure yields the set of $2 p$ equations

$$
\sum_{i=1}^{p} n_{i} A_{i}^{n}=B_{n} \quad(n=1,2, \ldots, 2 p)
$$

for $2 p$ parameters $n_{i}$ and $A_{i}$, with the right-hand sides

$$
B_{n} \equiv \frac{(-1)^{n-1}}{(n-1) !} \lim _{x \rightarrow 0} \frac{d^{n}}{d x^{n}} \ln f_{k}(x) .
$$

As is evident, the quantities $n_{i}, A_{i}$, as well as $B_{n}$, depend on the considered order $k$. But for avoiding excessively cumbersome notations, we do not use here the double labelling. When $k=2 p+1$ is odd, the odd-term factor approximant is

$$
f_{2 p+1}^{*}(x)=\prod_{i=1}^{p+1}\left(1+A_{i} x\right)^{n_{i}},
$$

with the parameters $A_{i}$ and $n_{i}$ defined by the re-expansion procedure yielding the set of equations

$$
\sum_{i=1}^{p+1} n_{i} A_{i}^{n}=B_{n} \quad(n=1,2, \ldots, 2 p+1),
$$

with the scaling condition $A_{1}=1$.

In this way, for any given expansion (1), the construction of self-similar factor approximants (2) or (4) is rather simple and straightforward. It has been shown [30-33] that the factor approximants are more general and accurate than Padé approximants, having, in addition, a principal advantage of being uniquely defined. This means that for each given order $k$ of expansion (1) there is just the sole factor approximant, while for each $k$ there exists a table of $k$ different Padé approximants $P_{[M / N]}$, with $M+N=k$. There is no general recipe for choosing one of the $k$ available Padé approximants. One often chooses the diagonal ones, but, as is easy to show, the latter are not always the most accurate ones. Such a problem of multiple possibilities does not arise for factor approximants: for each $k$, of the expansion $f_{k}(x)$, there is just one factor approximant $f_{k}^{*}(x)$.

\section{Exponents for $\mathrm{O}(\mathrm{N})$-symmetric theory}

Let us consider the $N$-component vector $\varphi^{4}$ field theory, for which the critical exponents can be obtained in the form of the $\varepsilon$-expansions, with $\varepsilon \equiv 4-d$, and $d$ being the space dimensionality. The derivation of these dimensional expansions can be found in the book [6]. In the Appendix A, we give the expansions that are considered in the present section. As is known, such expansions are divergent and require a resummation procedure. To illustrate more explicitly how the method of self-similar factor approximants works, let us start with the scalar singlecomponent field $(N=1)$. Then we have

$$
\begin{aligned}
\eta & \simeq 0.0185 \varepsilon^{2}+0.0187 \varepsilon^{3}-0.0083 \varepsilon^{4}+0.0257 \varepsilon^{5}, \\
\nu^{-1} & \simeq 2-0.333 \varepsilon-0.117 \varepsilon^{2}+0.124 \varepsilon^{3}-0.307 \varepsilon^{4}+0.951 \varepsilon^{5}, \\
\omega & \simeq \varepsilon-0.63 \varepsilon^{2}+1.62 \varepsilon^{3}-5.24 \varepsilon^{4}+20.75 \varepsilon^{5} .
\end{aligned}
$$

We reduce each of these expansions to the form

$$
f_{k}(\varepsilon)=f_{0}(\varepsilon) \sum_{n=0}^{k} a_{n} \varepsilon^{n}
$$

where $a_{0}=1$. According to Section 2, we construct the factor approximants

$$
f_{k}^{*}(\varepsilon)=f_{0}(\varepsilon) \prod_{i=1}^{N_{k}}\left(1+A_{i} \varepsilon\right)^{n_{i}}
$$

in which

$$
N_{k}= \begin{cases}\frac{k}{2}, & k=2 p=2,4, \ldots \\ \frac{k+1}{2}, & k=2 p+1=3,5, \ldots\end{cases}
$$

and the parameters $A_{i}$ and $n_{i}$ are obtained from the reexpansion procedure. Setting $\varepsilon=1$, we find the desired approximation $f_{k}^{*}(1) \equiv f_{k}^{*}$. The error bar for the approximant $f_{k}^{*}$ is given by

$$
\pm \frac{1}{2}\left(f_{k}^{*}-f_{k-1}^{*}\right) \quad k=2,3, \ldots
$$

Reducing the series for $\eta$ to form (7), we have $\eta_{0}(\varepsilon)=$ $0.0185 \varepsilon^{2}$. Constructing the factor approximants (8), we find for $\eta_{2}^{*}(\varepsilon)$ the parameters

$$
A_{1}=1.898511, \quad n_{1}=0.532423,
$$


and for $\eta_{3}^{*}(\varepsilon)$, we get

$A_{1}=1, n_{1}=0.789878, A_{2}=5.110862, n_{2}=0.043228$.

Setting $\varepsilon=1$, we obtain

$$
\eta_{2}^{*}=0.032602, \quad \eta_{3}^{*}=0.034588 .
$$

Thus, we conclude that the factor approximants give

$$
\eta=0.035 \pm 0.001
$$

In the same way, we proceed with the series for $\nu^{-1}$. In the second order, we find

$$
A_{1}=-0.869203, \quad n_{1}=-0.191555 .
$$

For the third order, we have

$A_{1}=1, n_{1}=-0.152346, A_{2}=0.023910, n_{2}=13.335389$.

In the fourth order, we get

$$
\begin{gathered}
A_{1}=3.027805, \quad n_{1}=-0.006791 \\
A_{2}=-0.440821, \quad n_{2}=-0.424352
\end{gathered}
$$

And for the fifth order, we find

$$
\begin{gathered}
A_{1}=1, \quad n_{1}=-0.045336, \\
A_{2}=4.168053, \quad n_{2}=-0.001772, \\
A_{3}=-0.312951, \quad n_{3}=-0.700494 .
\end{gathered}
$$

Setting $\varepsilon=1$, for the factor approximants $\nu_{k}^{*}$, we obtain

$$
\begin{array}{ll}
\nu_{2}^{*}=0.738227, & \nu_{3}^{*}=0.616528, \\
\nu_{4}^{*}=0.633852, & \nu_{5}^{*}=0.628417 .
\end{array}
$$

Hence, the result is

$$
\nu=0.628 \pm 0.003
$$

Finally, reducing the series for $\omega$ to form (7), we have $\omega_{0}(\varepsilon)=\varepsilon$. Following the standard procedure, for the factor approximant $\omega_{2}^{*}(\varepsilon)$, we get

$$
A_{1}=4.512857, \quad n_{1}=-0.139601 .
$$

For $\omega_{3}^{*}(\varepsilon)$, we find

$A_{1}=1, n_{1}=0.006238, A_{2}=4.547986, n_{2}=-0.137151$.

And for $\omega_{4}^{*}(\varepsilon)$, it follows

$$
\begin{gathered}
A_{1}=4.511659, \quad n_{1}=-0.139637, \\
A_{2}=107.494872, \quad n_{2}=-0.7 \times 10^{-7} .
\end{gathered}
$$

Setting $\varepsilon=1$, we obtain

$$
\omega_{2}^{*}=0.787958, \quad \omega_{3}^{*}=0.787160, \quad \omega_{4}^{*}=0.787934 .
$$

Hence, we come to the value

$$
\omega=0.788 \pm 0.0004 \text {. }
$$

Other critical exponents can be obtained from the scaling relations

$\alpha=2-\nu d, \beta=\frac{\nu}{2}(d-2+\eta), \gamma=\nu(2-\eta), \delta=\frac{d+2-\eta}{d-2+\eta}$,

which for the dimensionality $d=3$ simplifies to

$$
\alpha=2-3 \nu, \beta=\frac{\nu}{2}(1+\eta), \gamma=\nu(2-\eta), \delta=\frac{5-\eta}{1+\eta}
$$

Using here the found results for the factor approximants, we have

$$
\begin{array}{ll}
\alpha=0.116 \pm 0.009, & \beta=0.325 \pm 0.002, \\
\gamma=1.234 \pm 0.005, & \delta=4.797 \pm 0.006 .
\end{array}
$$

Accomplishing in the same way calculations for the arbitrary number of components $N$, we obtain the factor approximants for the critical exponents using the general series from the Appendix A. Our results are presented in Table 1. It is worth emphasizing that in the two limiting cases of $N=-2$ and $N=\infty$, where the exact critical exponents are known, our results coincide with these exact values. For $N=-2$, the exact exponents are

$$
\alpha=\frac{1}{2}, \beta=\frac{1}{4}, \gamma=1, \delta=5, \eta=0, \nu=\frac{1}{2} \quad(N=-2)
$$

in any dimension. And in the limit of large $N$, the exact exponents are

$$
\begin{gathered}
\alpha=\frac{d-4}{d-2}, \quad \beta=\frac{1}{2}, \quad \gamma=\frac{2}{d-2}, \quad \delta=\frac{d+4}{d-2}, \\
\eta=0, \quad \nu=\frac{1}{d-2}, \quad \omega=4-d \quad(N \rightarrow \infty),
\end{gathered}
$$

where $d$ is dimensionality. In three dimensions, the latter transforms to

$$
\alpha=-1, \beta=\frac{1}{2}, \gamma=2, \delta=5, \eta=0, \nu=1, \omega=1 .
$$

Since our results tend to the exact values when $N \rightarrow \infty$, the error bars diminish for $N \gg 1$, tending to zero, as $N \rightarrow \infty$. Thus, for $N=100$, the error bar is $10^{-2}$, for $N=1000$ it is $10^{-3}$, and for $N=10^{4}$, the error bar is $10^{-4}$. The error bars for $N \gg 10$ diminish as $1 / N$. The error bars for the factor approximants, up to $N=10$, obtained from the expansions for $\eta, \nu$, and $\omega$, are shown in Table 2.

Critical exponents have been calculated by Monte Carlo simulations [18-29,34-39] and other complicated numerical methods, as is reviewed in references $[6,17,40-42]$. Our results in Table 1 are in very good agreement with all these calculations. The advantage of our method is its simplicity. We have used only the expansions from the Appendix A. We do not need to know the large-order behavior of $\varepsilon$-expansions, which is required for other methods. 
Table 1. Critical exponents for the $N$-component $\varphi^{4}$ field theory, obtained by the summation of $\varepsilon$-expansions using the method of self-similar factor approximants.

\begin{tabular}{cccccccc}
\hline$N$ & $\alpha$ & $\beta$ & $\gamma$ & $\delta$ & $\eta$ & $\nu$ & $\omega$ \\
\hline-2 & 0.5 & 0.25 & 1 & 5 & 0 & 0.5 & 0.80118 \\
-1 & 0.36844 & 0.27721 & 1.07713 & 4.88558 & 0.019441 & 0.54385 & 0.79246 \\
0 & 0.24005 & 0.30204 & 1.15587 & 4.82691 & 0.029706 & 0.58665 & 0.78832 \\
1 & 0.11465 & 0.32509 & 1.23517 & 4.79947 & 0.034578 & 0.62854 & 0.78799 \\
2 & -0.00625 & 0.34653 & 1.31320 & 4.78962 & 0.036337 & 0.66875 & 0.78924 \\
3 & -0.12063 & 0.36629 & 1.38805 & 4.78953 & 0.036353 & 0.70688 & 0.79103 \\
4 & -0.22663 & 0.38425 & 1.45813 & 4.79470 & 0.035430 & 0.74221 & 0.79296 \\
5 & -0.32290 & 0.40033 & 1.52230 & 4.80254 & 0.034030 & 0.77430 & 0.79492 \\
6 & -0.40877 & 0.41448 & 1.57982 & 4.81160 & 0.032418 & 0.80292 & 0.79694 \\
7 & -0.48420 & 0.42676 & 1.63068 & 4.82107 & 0.030739 & 0.82807 & 0.79918 \\
8 & -0.54969 & 0.43730 & 1.67508 & 4.83049 & 0.029074 & 0.84990 & 0.80184 \\
9 & -0.60606 & 0.44627 & 1.71352 & 4.83962 & 0.027463 & 0.86869 & 0.80515 \\
10 & -0.65432 & 0.45386 & 1.74661 & 4.84836 & 0.025928 & 0.88477 & 0.80927 \\
50 & -0.98766 & 0.50182 & 1.98402 & 4.95364 & 0.007786 & 0.99589 & 0.93176 \\
100 & -0.89650 & 0.48334 & 1.92981 & 4.99264 & 0.001229 & 0.96550 & 0.97201 \\
1000 & -0.99843 & 0.49933 & 1.99662 & 4.99859 & 0.000235 & 0.99843 & 0.99807 \\
10000 & -0.99986 & 0.49993 & 1.99966 & 4.99986 & 0.000024 & 0.99984 & 0.99979 \\
$\infty$ & -1 & 0.5 & 2 & 5 & 0 & 1 & 1 \\
\hline
\end{tabular}

Table 2. Error bars for the critical exponent of table 1. For large $N \gg 10$, the error bars diminish with $N$ as $1 / N$.

\begin{tabular}{cccc}
\hline$N$ & $\eta$ error bar & $\nu$ error bar & $\omega$ error bar \\
\hline-2 & 0 & 0 & 0.0280 \\
-1 & 0.0007 & 0.0008 & 0.0013 \\
0 & 0.0010 & 0.0018 & 0.0110 \\
1 & 0.0010 & 0.0027 & 0.0043 \\
2 & 0.0009 & 0.0034 & 0.0016 \\
3 & 0.0008 & 0.0038 & 0.0020 \\
4 & 0.0007 & 0.0039 & 0.0016 \\
5 & 0.0006 & 0.0038 & 0.0007 \\
6 & 0.0005 & 0.0036 & 0.0005 \\
7 & 0.0004 & 0.0034 & 0.0019 \\
8 & 0.0003 & 0.0032 & 0.0032 \\
9 & 0.0002 & 0.0030 & 0.0042 \\
10 & 0.0001 & 0.0029 & 0.0048 \\
\hline
\end{tabular}

\section{Exponents for spin glass}

Here we show that the method of self-similar factor approximants can be applied to such a notoriously difficult problem as summing the high-temperature series of the Ising spin glass. This model is described by the Hamiltonian

$$
H=-\sum_{(i j)} J_{i j} \sigma_{i} \sigma_{j},
$$

in which $(i j)$ implies the summation over nearest neighbors, $\sigma_{i}$ takes values \pm 1 , and $J_{i j}$ are independent random variables, whose dimensionless forms $\bar{J}_{i j} \equiv \beta J_{i j}$, with $\beta$ being inverse temperature, occur with the probability

$$
p\left(\bar{J}_{i j}\right)=\frac{1}{2}\left[\delta\left(\bar{J}_{i j}-\bar{J}\right)+\delta\left(\bar{J}_{i j}+\bar{J}\right)\right]
$$

where $\bar{J}_{i j}=\beta J$ is a parameter. Monte Carlo simulations $[43,34,45]$ demonstrate the existence of the phase transition in three dimensions.

The phase transition corresponds to a singularity in susceptibilities. One considers two types of the latter, the Edwards-Anderson susceptibility

$$
\chi_{E A} \equiv \frac{1}{N} \sum_{i, j}\left\langle\left\langle\left(\left\langle\sigma_{i} \sigma_{j}\right\rangle^{2}\right)\right\rangle\right\rangle,
$$

and the auxiliary susceptibility

$$
\chi^{\prime} \equiv \frac{1}{N} \sum_{i, j}\left[\left\langle\left\langle\left(\left\langle\sigma_{i} \sigma_{j}\right\rangle^{2}\right)\right\rangle\right\rangle\right]^{2} .
$$

Here $N$ is the total number of lattice sites, single angular brackets $\langle\ldots\rangle$ refer to thermal averaging, and the double brackets $\langle\langle\ldots\rangle\rangle$ refer to averaging with respect to the distribution of interactions, defined by probability (12). When temperature $T$ approaches the critical temperature $T_{c}$, susceptibilities (13) and (14) behave as

$$
\chi_{E A} \propto\left(T-T_{c}\right)^{-\gamma}, \quad \chi^{\prime} \propto\left(T-T_{c}\right)^{-\gamma^{\prime}} .
$$

High-temperature series expansions for susceptibilities (13) and (14) are represented as series in powers of

$$
w \equiv \tanh ^{2}(\beta J) .
$$

Analyzing the series

$$
\chi_{E A} \simeq \sum_{n} a_{n} w^{n}, \quad \chi^{\prime} \simeq \sum_{n} a_{n}^{\prime} w^{n}
$$


one aims at finding the critical exponents $\gamma$ and $\gamma^{\prime}$ characterizing the critical behavior (15). These exponents are connected with each other through the scaling relations

$$
\gamma=(2-\eta) \nu, \quad \gamma^{\prime}=(4-d-2 \eta) \nu,
$$

where $\eta$ and $\nu$ are the critical exponent defining the behavior of the correlation function $\left\langle\left\langle\left(\left\langle\sigma_{i} \sigma_{j}\right\rangle^{2}\right)\right\rangle\right\rangle$ and the correlation length $\xi \propto\left(T-T_{c}\right)^{-\nu}$, and where $d$ is dimensionality. Therefore, the exponents $\gamma$ and $\gamma^{\prime}$ can be expressed one through another by means of the scaling relation

$$
2 \gamma=\gamma^{\prime}+\nu d
$$

provided $\nu$ is known.

The analysis of expansions (17) turned out to be extremely difficult. This is because the first few terms of the series contain little information on spin-glass ordering. Actually, the coefficients of the first three terms of the series for $\chi_{E A}$ are identical to those for the susceptibility series of the pure Ising model. In fact, one cannot see any spin-glass behavior until one gets contribution from higher orders. This happens because an essential feature of spin glass is frustration, which reveals itself only in higher orders of the series. Hence, any analysis, depending sensitively on the first few terms in determining the critical exponents $\gamma$ and $\gamma^{\prime}$, is not likely to give correct answers. These difficulties have been described in detail by Singh and Chakravarty [46,47], who found that more than ten terms in expansions (13) and (14) are necessary to be able to estimate the critical exponents $\gamma$ and $\gamma^{\prime}$. They derived [46] in three dimensions expansions for $\chi_{E A}$ and $\chi^{\prime}$ up to 17th order and in four dimensions, an expansion for $\chi_{E A}$ up to 15 th order.

However, even having quite a number of terms in expansions (17), it is very difficult to find the related critical exponents. Since, as is stressed above, the effects of frustration reveal themselves only in high orders of expansions, so that the lower orders do not provide correct information on spin glass behavior. The method that has been found [47] to be most suitable to this problem is that of inhomogeneous differential approximants, which is a generalization of the $d$-log Padé summation. A weak point of this method is that the approximants, for each given order $k$ of an expansion, are not uniquely defined. Thus, for an expansion of order $k=10$, there are 42 variants of the approximants, for the expansion of order $k=15$, there are 96 variants, and for the order $k=17$, one has 136 variants of different approximants. All these various approximants yield the results that are quite different from each other, and it is not clear which of them are to be accepted as correct and which as wrong, so that the problem arises of a subjective, not strictly defined, choice of some of them labelled as "well-behaved".

Now let us apply to expansions (17) the method of self-similar factor approximants of Section 2. In three dimensions $(d=3)$, both series for $\chi_{E A}$ as well as for $\chi^{\prime}$ are known [47] up to seventeenth order. The effect of frustration, typical of spin glass, occurs in the series for $\chi^{\prime}$ much earlier than in that for $\chi_{E A}$. Therefore more accurate results can be obtained considering the series for $\chi^{\prime}$, which are

$$
\begin{gathered}
\chi^{\prime}=1+6 w^{2}+102 w^{4}-192 w^{5}+1998 w^{6}-7584 w^{7} \\
+42822 w^{8}-221856 w^{9}+1147878 w^{10}-5980608 w^{11} \\
+32318910 w^{12}-167464128 w^{13}+906131742 w^{14} \\
-4849958304 w^{15}+25952889798 w^{16}-141648771168 w^{17} .
\end{gathered}
$$

For these series, we construct the factor approximants following the standard procedure of Section 2. The closest singularity to the origin defines the critical points $w_{c}$ and the related critical exponents $\gamma^{\prime}$. For the series of order $k=15$, we find $w_{c}=0.42$ and $\gamma^{\prime}=2.07$; for $k=16$, we have $w_{c}=0.39$ and $\gamma^{\prime}=1.44$; and for $k=17$, we get $w_{c}=0.41$ with $\gamma^{\prime}=1.82$. Thus, for the critical exponent $\gamma^{\prime}$, we obtain

$$
\gamma^{\prime}=1.82 \pm 0.19 \quad(d=3) .
$$

This can be compared with the results of the Monte Carlo simulations [45], which find the phase transition at $T_{c}=$ $1.18 \pm 0.03$, with the critical exponent

$$
\gamma_{M C}^{\prime}=1.87 \pm 0.28 \quad(d=3) .
$$

Using our result (21), the known value [40] of $\nu=1.3 \pm 0.1$, and the scaling relation (19), we find the critical exponent

$$
\gamma=2.86 \pm 0.24 \quad(d=3) .
$$

From the Monte Carlo simulations [40] it follows

$$
\gamma_{M C}=2.89 \pm 0.29 \quad(d=3)
$$

As is seen, the critical exponents in equations (21) and (23) are close to the Monte Carlos values in equations (32) and (24), respectively.

In four dimensions $(d=4)$, only the series of fifteenth order for $\chi_{E A}$ are available [47], which are

$$
\begin{gathered}
\chi=1+8 w+56 w^{2}+392 w^{3}+2408 w^{4}+15272 w^{5}+85352 w^{6} \\
+508808 w^{7}+2625896 w^{8}+15111976 w^{9}+72067672 w^{10} \\
+421464680 w^{11}+1851603192 w^{12}+11810583208 w^{13} \\
+46346625320 w^{14}+347729503368 w^{15}
\end{gathered}
$$

Constructing the factor approximants for these series, in the highest orders we find $w_{c}=0.20$, with $\gamma=1.59$, for $k=14$ and $w_{c}=0.21$, with $\gamma=2.35$, for $k=15$. Therefore, for the critical exponent $\gamma$, we obtain

$$
\gamma=2.35 \pm 0.38 \quad(d=4) .
$$

To our knowledge, Monte Carlo simulations for $d=4$ are not available. And the method of inhomogeneous differential approximants [47] estimates $\gamma \approx 2.0 \pm 0.4$. Since there is neither an expansion for $\chi^{\prime}$ nor information on other indices, it is not possible to determine the exponent $\gamma^{\prime}$ in $d=4$.

The example of the present section shows that the method of self-similar factor approximants can be applied to rather complicated series with very nontrivial behavior, requiring the consideration of high-order terms. 


\section{Conclusion}

The method of self-similar factor approximants [30-33] is applied to calculating the critical exponents of the $N$ component vector $\varphi^{4}$ field theory and of the Ising spin glass. The first example is chosen because of the wide interest to the $O(N)$-symmetric $\varphi^{4}$ theory, which serves as a typical model for characterizing the critical behavior of a large variety of physical systems. We showed that the application of the method to $\varepsilon$-expansions is very simple and straightforward at the same time providing the accuracy comparable with that of other essentially more complicated techniques.

The case of high-temperature expansions for the Ising spin glass is taken as an example of series with a notoriously nontrivial structure, requiring the consideration of high-order terms and making it very difficult an unambiguous determination of the exponents for susceptibilities by other known methods. Our method allows us to find the exponents that are in good agreement with Monte Carlo simulations, when the latter are available.

In the present paper, we have concentrated on the calculations of critical exponents. Of course, determining the critical points is also of importance. For instance, recently there has been a great interest to an accurate calculation of the critical temperature $T_{c}$ for interacting Bose gas (see review articles $[48,49])$. The most accurate results have been obtained so far by using the ideas of the optimized perturbation theory [5] in references [50-55] and by employing Monte Carlo simulations [56-60]. These are rather involved numerical techniques. The method of self-similar factor approximants can also be applied to this problem, which, however, is a topic for a separate investigation.

\section{Appendix A}

The derivation of the expansions for the $N$-component field theory, considered in Section 2, can be found in the book [6]. These expansions are

$$
\begin{gathered}
\eta(\varepsilon)=\frac{(N+2) \varepsilon^{2}}{2(N+8)^{2}}\left\{1+\frac{\varepsilon}{4(N+8)^{2}}\left[-N^{2}+56 N+272\right]\right. \\
-\frac{\varepsilon^{2}}{16(N+8)^{4}}\left[5 N^{4}+230 N^{3}-1124 N^{2}-17920 N\right. \\
-46144+384 \zeta(3)(N+8)(5 N+22)]-\frac{\varepsilon^{3}}{64(N+8)^{6}}\left[13 N^{6}\right. \\
+946 N^{5}+27620 N^{4}+121472 N^{3}-262528 N^{2}-2912768 N \\
-5655552-16 \zeta(3)(N+8)\left(N^{5}+10 N^{4}+1220 N^{3}\right. \\
\left.-1136 N^{2}-68672 N-171264\right)+1152 \zeta(4)(N+8)^{3}(5 N+22) \\
\left.\left.-5120 \zeta(5)(N+8)^{2}\left(2 N^{2}+55 N+186\right)\right]\right\}
\end{gathered}
$$

$$
\begin{aligned}
& \nu^{-1}=2+\frac{(N+2) \varepsilon}{N+8}\left\{-1-\frac{\varepsilon}{2(N+8)^{2}}[13 N+44]\right. \\
& +\frac{\varepsilon^{2}}{8(N+8)^{4}}\left[3 N^{3}-452 N^{2}-2672 N-5312\right. \\
& +96 \zeta(3)(N+8)(5 N+22)]+\frac{\varepsilon^{3}}{8(N+8)^{6}}\left[3 N^{5}+398 N^{4}\right. \\
& -12900 N^{3}-81552 N^{2}-219968 N-357120 \\
& +16 \zeta(3)(N+8)\left(3 N^{4}-194 N^{3}+148 N^{2}+9472 N+19488\right) \\
& +288 \zeta(4)(N+8)^{3}(5 N+22)-1280 \zeta(5)(N+8)^{2}\left(2 N^{2}\right. \\
& +55 N+186)]+\frac{\varepsilon^{4}}{128(N+8)^{8}}\left[3 N^{7}-1198 N^{6}-27484 N^{5}\right. \\
& -1055344 N^{4}-5242112 N^{3}-5256704 N^{2}+6999040 N \\
& -626688-16 \zeta(3)(N+8)\left(13 N^{6}-310 N^{5}+19004 N^{4}\right. \\
& \left.+102400 N^{3}-381536 N^{2}-2792576 N-4240640\right) \\
& -1024 \zeta^{2}(3)(N+8)^{2}\left(2 N^{4}+18 N^{3}+981 N^{2}+6994 N\right. \\
& +11688)+48 \zeta(4)(N+8)^{3}\left(3 N^{4}-194 N^{3}+148 N^{2}\right. \\
& +9472 N+19488)+256 \zeta(5)(N+8)^{2}\left(155 N^{4}+3026 N^{3}\right. \\
& \left.+989 N^{2}-66018 N-130608\right)-6400 \zeta(6)(N+8)^{4}\left(2 N^{2}\right. \\
& +55 N+186)+56448 \zeta(7)(N+8)^{3}\left(14 N^{2}\right. \\
& +189 N+256)]\} \\
& \omega(\varepsilon)=\varepsilon-\frac{3 \varepsilon^{2}}{(N+8)^{2}}[3 N+14] \\
& +\frac{\varepsilon^{3}}{4(N+8)^{4}}\left[33 N^{3}+538 N^{2}+4288 N+9568\right. \\
& +96 \zeta(3)(N+8)(5 N+22)]+\frac{\varepsilon^{4}}{16(N+8)^{6}}\left[5 N^{5}\right. \\
& -1488 N^{4}-46616 N^{3}-419528 N^{2}-1750080 N-2599552 \\
& -96 \zeta(3)(N+8)\left(63 N^{3}+548 N^{2}+1916 N+3872\right) \\
& +288 \zeta(4)(N+8)^{3}(5 N+22)-1920 \zeta(5)(N+8)^{2}\left(2 N^{2}\right. \\
& +55 N+186)]+\frac{\varepsilon^{5}}{64(N+8)^{8}}\left[13 N^{7}+7196 N^{6}+240328 N^{5}\right. \\
& +3760776 N^{4}+38877056 N^{3}+223778048 N^{2}+660389888 N \\
& +752420864-16 \zeta(3)(N+8)\left(9 N^{6}-1104 N^{5}-11648 N^{4}\right. \\
& \left.-243864 N^{3}-2413248 N^{2}-9603328 N-14734080\right) \\
& -768 \zeta^{2}(3)(N+8)^{2}\left(6 N^{4}+107 N^{3}+1826 N^{2}+9008 N\right. \\
& +8736)-288 \zeta(4)(N+8)^{3}\left(63 N^{3}+548 N^{2}+1916 N+3872\right) \\
& +256 \zeta(5)(N+8)^{2}\left(305 N^{4}+7386 N^{3}+45654 N^{2}+143212 N\right. \\
& +226992)-9600 \zeta(6)(N+8)^{4}\left(2 N^{5}+55 N+186\right) \\
& \left.+112896 \zeta(7)(N+8)^{3}\left(14 N^{2}+189 N+256\right)\right] \text {. }
\end{aligned}
$$

Here $\varepsilon=4-d$ is assumed to be asymptotically small, $\varepsilon \rightarrow 0$. 


\section{References}

1. H.E. Stanley, Introduction to Phase Transitions and Critical Phenomena (Oxford University, New York, 1987)

2. V.I. Yukalov, A.S. Shumovsky, Lectures on Phase Transitions (World Scientific, Singapore, 1990)

3. D. Sornette, Critical Phenomena in Natural Sciences (Springer, Berlin, 2006)

4. J. Zinn-Justin, Quantum Field Theory and Critical Phenomena (Oxford Univeristy, Oxford, 1996)

5. V.I. Yukalov, Moscow Univ. Phys. Bull. 31, 10 (1976)

6. H. Kleinert, V. Schulte-Frohlinde, Critical Properties of $\varphi^{4}$-Theories (World Scientific, Singapore, 2006)

7. H. Kleinert, Path Integrals (World Scientific, Singapore, 2006)

8. V.I. Yukalov, Phys. Rev. A 42, 3324 (1990)

9. V.I. Yukalov, Physica A 167, 833 (1990)

10. V.I. Yukalov, J. Math. Phys. 32, 1235 (1991)

11. V.I. Yukalov, J. Math. Phys. 33, 3994 (1992)

12. V.I. Yukalov, E.P. Yukalova, Physica A 225, 336 (1996)

13. V.I. Yukalov, S. Gluzman, Phys. Rev. Lett. 79, 333 (1997)

14. S. Gluzman, V.I. Yukalov, Phys. Rev. E 55, 3983 (1997)

15. V.I. Yukalov, E.P. Yukalova, Ann. Phys. 277, 219 (1999)

16. V.I. Yukalov, E.P. Yukalova, Chaos Solitons Fractals 14 $839(2002)$

17. H. Kleinert, V.I. Yukalov, Phys. Rev. E 71, 026131 (2005)

18. H.W. Blöte, A. Compagner, J.H. Croockewit, Y.T. Fonk, J.R. Heringa, A. Hoogland, T.S. Smit, A.L. van Villingen, Physica A 161, 1 (1989)

19. W. Janke, Phys. Lett. A 148, 306 (1990)

20. A.M. Ferrenberg, D.P. Landau, Phys. Rev. B 44, 5081 (1991)

21. C.F. Baillie, R. Gupta, K.A. Hawick, G.S. Pawley, Phys. Rev. B 45, 10438 (1992)

22. C. Holm, W. Janke, Phys. Lett. A 173, 8 (1993)

23. C. Holm, W. Janke, Phys. Rev. B 48, 936 (1993)

24. K. Chen, A.M. Ferrenberg, D.P. Landau, Phys. Rev. B 48, 3249 (1993)

25. C. Holm, W. Janke, J. Appl. Phys. 73, 5488 (1993)

26. B. Li, N. Madras, A.D. Sokal, J. Stat. Phys. 80, 661 (1995)

27. K. Kanaya, S. Kaya, Phys. Rev. D 51, 2404 (1995)

28. H.G. Ballesteros, L.A. Fernandez, V. Martin-Mayor, A.M. Sudupe, Phys. Lett. B 387, 125 (1996)

29. S. Caracciolo. M.S. Causo, A. Pelisseto, Phys. Rev. E 57, 1215 (1998)
30. V.I. Yukalov, S. Gluzman, D. Sornette, Physica A 328, 409 (2003)

31. S. Gluzman, V.I. Yukalov, D. Sornette, Phys. Rev. E 67, 026109 (2003)

32. V.I. Yukalov, S. Gluzman, Int. J. Mod. Phys. B 18, 3027 (2004)

33. V.I. Yukalov, E.P. Yukalova, Phys. Rev. A 72, 063611 (2005)

34. D.P. Landau, J. Magn. Magn. Mater. 200, 231 (1999)

35. M. Hasenbusch, J. Phys. A 34, 8221 (2001)

36. M. Campostrini, M. Hasenbusch, A. Pelissetto, P. Rossi, E. Vicari, Phys. Rev. B 65, 144520 (2002)

37. Y. Deng, H.W. Blöte, Phys. Rev. E 68, 036125 (2003)

38. M. Hasenbusch, A. Pelissetto, E. Vicari, Phys. Rev. B 72, 014532 (2005)

39. M. Campostrini, M. Hasenbusch, A. Pelissetto, E. Vicari, Phys. Rev. B 74, 144506 (2006)

40. R. Guida, J. Zinn-Justin, J. Phys. A 31, 8103 (1998)

41. J. Zinn-Justin, Phys. Rep. 344, 159 (2001)

42. A. Pelissetto, E. Vicari, Phys. Rep. 368, 549 (2002)

43. R.N. Bhatt, A.P. Young, Phys. Rev. Lett. 54, 924 (1985)

44. A.T. Ogielski, I. Morgenstern, Phys. Rev. Lett. 54, 928 (1985)

45. A.T. Ogielski, Phys. Rev. B 32, 7384 (1985)

46. R.R.P. Singh, S. Chakravarty, Phys. Rev. B 36, 546 (1987)

47. R.R.P. Singh, S. Chakravarty, Phys. Rev. B 36, 559 (1987)

48. J.O. Andersen, Rev. Mod. Phys. 76, 599 (2004)

49. V.I. Yukalov, Laser Phys. Lett. 1, 435 (2004)

50. B. Kastening, Phys. Rev. A 68, 061601 (2003)

51. B. Kastening, Laser Phys. 14, 586 (2004)

52. B. Kastening, Phys. Rev. A 69, 043613 (2004)

53. J.L. Kneur, A. Neveu, M.B. Pinto, Phys. Rev. A 69, 053624 (2004)

54. B. Kastening, Phys. Rev. A 70, 043621 (2004)

55. J.L. Kneur, M.B. Pinto, Phys. Rev. A 71, 033613 (2005).

56. P. Arnold, G. Moore, Phys. Rev. Lett. 87, 120401 (2001)

57. V.A. Kashurnikov, N.V. Prokofiev, B.V. Svistunov, Phys. Rev. Lett. 87, 120402 (2001)

58. P. Arnold, G. Moore, Phys. Rev. E 64, 066113 (2001)

59. N.V. Prokofiev, B.V. Svistunov, Phys. Rev. Lett. 87, 160601 (2001)

60. K. Nho, D.P. Landau, Phys. Rev. A 70, 053614 (2004) 